MARia Ekes (Warszawa)

\title{
CORE AND EQUILIBRIA IN MODELS OF LARGE HOUSEHOLD ECONOMY
}

Abstract. The paper deals with models of household economy with infinitely many agents classified into a finite number of types. The notions of competitive equilibrium, core and quasi-core are examined with special emphasis on their mutual relations.

The paper presents the discussion of relations between the core and equilibria in models of economy with infinitely many agents classified into a finite number of different types and with some elements of labor market (see Ekes [3], Wieczorek [8], [9]). By "infinitely many agents" we understand a continuum of agents. We do not introduce any structure on the set of agents (i.e. nonatomic measure space) since we are interested only in distributions of agents' decisions and agents' consumption - we apply here an anonymous approach (see e.g. Mas-Colell [5]). Agents are first considered as producersthey make a choice among a certain number of activities resulting in the production of chosen goods. Agents of the same type are characterized by their capacities to perform each kind of activity. The capacities are expressed by nonnegative numbers interpreted as efficiency coefficients of agents of a given type while choosing the respective activities. They may correspond to their skills, education, technical possibilities etc. In some cases certain types may be unable to undertake some activities; then the corresponding coefficients are 0 . Agents are also considered as consumers: they are characterized by their demand function, depending on the individual income and the system of prevailing prices; the demand function is assumed to be the same for all agents of the same type but it may differ among the types. We are specially interested in situations where the demand functions are interpreted as solutions of the problem of maximization of utility under the

2000 Mathematics Subject Classification: 90A14, 90A40, 90A11.

Key words and phrases: household economy, competitive equilibrium, core, quasi-core. The research was supported by KBN grant \#5 H02B 00820. 
budget constraints. The labor is the only input in our model and hence we refer to the model as "household economy".

Models of competition with infinitely many agents are known in the literature since Aumann's celebrated papers [1], [2]. In [1] Aumann proved that in a pure exchange economy with a nonatomic space of agents the core and the set of competitive equilibria coincide, i.e. each competitive allocation is in the core and for each core allocation there exists a system of prices with which it forms a competitive equilibrium (analogous results were also obtained in Hildenbrand [4], Vind [7]). The situation in our model is analogous only to some extent, since the relations mentioned above apply to two related but different notions concerning the cooperative solution: core and quasi-core. In the following we formulate the model and state and prove the main results, Theorems 1 and 4 .

1. Elements of the model. The formal description of the model is given by the following elements:

- a positive integer $n$ : the number of types of agents; each type contains infinitely many individuals, the structure of the population of agents of each type is described by a vector $q=\left(q_{1}, \ldots, q_{n}\right)$ of positive reals;

- a positive integer $k$ : the number of different activities and of different commodities considered in the model;

- a system $\left(r_{j}^{i} \mid i=1, \ldots, n, j=1, \ldots, k\right)$ with $r_{j}^{i} \geq 0$ for all $i$ and $j$ : the system of efficiency coefficients, characterizing the efficiency of the agents; the number $r_{j}^{i}$ is interpreted as the volume of the production of the $j$ th commodity by an agent of type $i$ who has chosen the $j$ th activity;

- functions $d^{i}: \mathbb{R}_{+} \times \Delta_{k} \rightarrow \mathbb{R}_{+}^{k}$ for $i=1, \ldots, n$ : the demand functions of agents of respective types; the demand $d^{i}=d^{i}(I, \pi)$ depends on the individual income $I$ and the system of prevailing prices $\pi$ chosen from the standard simplex.

We assume that the value of the demand of an agent is equal to the income for all values of income and prices in their respective domains, i.e.

$$
\left\langle d^{i}(I, \pi) ; \pi\right\rangle=I
$$

for all $I \in \mathbb{R}_{+}$and $\pi \in \Delta_{k}(\langle\cdot ; \cdot\rangle$ denotes the inner product $)$. We will denote the household economy described above by $\mathfrak{E}$.

We say that a demand function $d: \mathbb{R}_{+} \times \Delta_{k} \rightarrow \mathbb{R}_{+}^{k}$ is adapted to the utility function $u: \mathbb{R}_{+}^{k} \rightarrow \mathbb{R}$ whenever

$$
d(I, \pi) \in \operatorname{Argmax}\left\{u(\mathbf{x}) \mid \mathbf{x} \in \mathbb{R}_{+}^{k},\langle\pi ; \mathbf{x}\rangle \leq I\right\}
$$

for all $I \in \mathbb{R}_{+}$and $\pi \in \Delta_{k}$, which means that the demand $d(I, \pi)$ maximizes the utility function $u$ under the budget constraint $\langle\pi ; \mathbf{x}\rangle \leq I$. We consider 
the situation where the demand functions $d^{i}$ are adapted to utility functions $u^{i}: \mathbb{R}_{+}^{k} \rightarrow \mathbb{R}$ for all $i=1, \ldots, n$.

For $\mathbf{x}, \mathbf{y} \in \mathbb{R}^{k}$ we shall write $\mathbf{x} \leq \mathbf{y}$ if $x_{i} \leq y_{i}$ for $i=1, \ldots, k ; \mathbf{x}<\mathbf{y}$ if $x_{i} \leq y_{i}$ for $i=1, \ldots, k$ but $\mathbf{x} \neq \mathbf{y}$; and $\mathbf{x} \ll \mathbf{y}$ if $x_{i}<y_{i}$ for $i=1, \ldots, k$. We shall also use the inverse inequalities $\geq,>, \gg$.

We say that a utility function $u: \mathbb{R}_{+}^{k} \rightarrow \mathbb{R}$ is essentially increasing whenever $u(\mathbf{y})<u(\mathbf{x})$ for $\mathbf{x}, \mathbf{y} \in \mathbb{R}_{+}^{k}$ such that $\mathbf{y} \ll \mathbf{x}$.

If the distribution of actions of agents of type $i$, after having decided what kind of work to perform, is $p^{i}\left(p_{j}^{i}\right.$ is the fraction of agents of type $i$ who produce the $j$ th commodity) and we denote by $\mathbf{p}=\left(p^{1}, \ldots, p^{n}\right)$ the vector of distributions of all types, then the aggregated supply is given by the formula

$$
S(\mathbf{p}):=\left(\sum_{i=1}^{n} q_{i} r_{1}^{i} p_{1}^{i}, \ldots, \sum_{i=1}^{n} q_{i} r_{k}^{i} p_{k}^{i}\right),
$$

while the aggregated demand is

$$
D(\mathbf{p}, \pi):=\sum_{i=1}^{n} q_{i} \sum_{j=1}^{k} d^{i}\left(r_{j}^{i} \pi_{j}, \pi\right) \cdot p_{j}^{i} .
$$

Note that the aggregated demand, unlike the aggregated supply, also depends on the prevailing prices.

We say that a vector $\mathbf{x} \in \mathbb{R}_{+}^{k}$ is Pareto optimal whenever there exists no vector $\mathbf{y} \in \mathbb{R}_{+}^{k}$ such that $\mathbf{y}=S(\mathbf{p})$ for some distribution $\mathbf{p}$ and $\mathbf{y}>\mathbf{x}$. We say that a vector $\mathbf{x} \in \mathbb{R}_{+}^{k}$ is weakly Pareto optimal whenever there exists no vector $\mathbf{y} \in \mathbb{R}_{+}^{k}$ such that $\mathbf{y}=S(\mathbf{p})$ for some distribution $\mathbf{p}$ and $\mathbf{y} \gg \mathbf{x}$. A distribution vector $\mathbf{p}$ is Pareto efficient (weakly Pareto efficient) whenever the aggregated supply $S(\mathbf{p})$ is Pareto optimal (weakly Pareto optimal).

A system $(\mathbf{p} ; \pi) \in\left(\Delta_{k}\right)^{n+1}$, consisting of the sequence of distributions of all agents' actions and a price system is at competitive equilibrium whenever it satisfies the following conditions:

$$
\begin{gathered}
r_{j}^{i} \pi_{j}=\max _{l=1, \ldots, k} r_{l}^{i} \pi_{l}, \quad \text { unless } p_{j}^{i}=0, \quad \text { for } i=1, \ldots, n, j=1, \ldots, k ; \\
D(\mathbf{p}, \pi) \leq S(\mathbf{p}) .
\end{gathered}
$$

Thus at equilibrium all agents of the same type have the same and maximal possible income (although they may choose different activities) and the aggregated demand is not greater than the aggregated supply.

It is important to note that the choice of the kind of work is crucial in our model because in this way agents are maximizing their income (thus their consumption) under prevailing prices.

2. Definitions of the core and quasi-core. Denote by $V:=\{1, \ldots, k\}$ the set of all activities and by $W:=V \times \mathbb{R}_{+}^{k}$ the Cartesian product of the set 
of activities and the set of all vectors of commodities. A state of the economy $\mathfrak{E}$ is a sequence $\mathbf{s}=\left(s^{1}, \ldots, s^{n}\right)$ of normed measures on Borel subsets of $W$. A state is thus an anonymous description of decisions taken by agents and of allocation of commodities among the agents of all types.

A state $\mathbf{s}$ is admissible if

$$
\sum_{i=1}^{n} q_{i} \int_{\mathbb{R}_{+}^{k}} \mathbf{x} s^{i}(V \times d \mathbf{x}) \leq\left(\sum_{i=1}^{n} q_{i} r_{1}^{i} s_{1}^{i}, \ldots, \sum_{i=1}^{n} q_{i} r_{k}^{i} s_{k}^{i}\right),
$$

where $s_{j}^{i}=s^{i}\left(\{j\} \times \mathbb{R}_{+}^{k}\right)$ for $i=1, \ldots, n$ and $j=1, \ldots, k$ (the notation $\int_{\mathbb{R}_{+}^{k}} \mathbf{x} s^{i}(V \times d \mathbf{x})$ means that we are integrating with respect to the marginal measure on $\mathbb{R}_{+}^{k}$ of the measure $s^{i}$ defined on the Cartesian product $V \times \mathbb{R}_{+}^{k}$; we shall use such notation in what follows). The condition means that the aggregated consumption is not greater than the total production.

Given a state $\mathbf{s}$, a coalition is understood as a system $\boldsymbol{\sigma}=\left(\sigma^{1}, \ldots, \sigma^{n}\right)$ consisting of measures $\sigma^{i}$ on Borel subsets of $W$ such that, for any Borel subset $A$ in $W, \sigma^{i}(A) \leq s^{i}(A)$ for $i=1, \ldots, n$. A coalition is nonzero whenever $\sigma^{i}(W)>0$ for at least one type $i$.

An action available to a coalition $\boldsymbol{\sigma}$ is a sequence $\mathbf{f}=\left(f^{1}, \ldots, f^{n}\right)$ of transition functions, defined on the Cartesian product $W \times \mathfrak{B}(W)\left(f^{i}\right.$ : $W \times \mathfrak{B}(W) \rightarrow[0,1]$, i.e. for each fixed $w \in W, f^{i}(w, \cdot)$ is a normalized measure on $\mathfrak{B}(W)$ while for each fixed Borel set $A \in \mathfrak{B}(W)$, the function $f^{i}(\cdot, A)$ is Borel measurable). The composition of a measure $\sigma$ on $\mathfrak{B}(W)$ with a transition function $f$ will be denoted by $m_{\mathbf{f}}=\sigma \circ \mathbf{f}$ (i.e. it is the unique measure on $\mathfrak{B}(W \times W)$ such that $m_{\mathbf{f}}(A \times B)=\int_{B} f(w, A) \sigma(d w)$ for all $A, B \in \mathfrak{B}(W))$. An action of a coalition is an anonymous description of changes of decisions made by the members of the coalition, concerning the undertaken activities and re-allocation of commodities within the coalition.

An action $\mathbf{f}$ is admissible to a coalition $\boldsymbol{\sigma}$ whenever only the aggregated production within the coalition is allocated to its members, i.e. when

$$
\sum_{i=1}^{n} q_{i} \int_{\mathbb{R}_{+}^{k}} \mathbf{x} m_{\mathbf{f}}^{i}\left(V \times \mathbb{R}_{+}^{k} \times V \times d \mathbf{x}\right) \leq\left(\sum_{i=1}^{n} q_{i} r_{1}^{i} m_{\mathbf{f}}^{i}\left(V_{1}\right), \ldots, \sum_{i=1}^{n} q_{i} r_{k}^{i} m_{\mathbf{f}}^{i}\left(V_{k}\right)\right),
$$

where we have set $V_{j}=V \times \mathbb{R}_{+}^{k} \times\{j\} \times \mathbb{R}_{+}^{k}$ for $j=1, \ldots, k$.

An action $\mathbf{f}$ is profitable to a coalition $\boldsymbol{\sigma}$ whenever its members choose other available activities and re-allocate commodities and so they (almost) all get an increase of their utility, i.e.

$$
m_{\mathbf{f}}^{i}\left(\left\{\left((j, \mathbf{x}),\left(j^{\prime}, \mathbf{x}^{\prime}\right)\right) \in W \times W \mid u^{i}\left(\mathbf{x}^{\prime}\right)>u^{i}(\mathbf{x})\right\}\right)=m_{\mathbf{f}}^{i}(W \times W)
$$

for $i=1, \ldots, n$ (as before, $m_{\mathbf{f}}^{i}$ denotes $\sigma^{i} \circ f^{i}$ ). 
The core of the economy $\mathfrak{E}$ is defined as the set of all its admissible states s such that there exists no nonzero coalition $\boldsymbol{\sigma}$ which has an admissible and profitable action $\mathbf{f}$.

The quasi-core of the economy $\mathfrak{E}$ is the set of all its admissible states $\mathbf{s}$ such that there exists no nonzero coalition $\boldsymbol{\sigma}$ which has an admissible and profitable action $\mathbf{f}$ satisfying, for $i=1, \ldots, n$,

$$
\sum_{j=1}^{k} m_{\mathbf{f}}^{i}\left(\{j\} \times \mathbb{R}_{+}^{k} \times\{j\} \times \mathbb{R}_{+}^{k}\right)=m_{\mathbf{f}}^{i}(W \times W) .
$$

The above condition states that the only result of an action $\mathbf{f}$ is a change of the allocation of commodities in the coalition; the decisions concerning the choice of produced commodities remain unchanged within the coalition.

Notice that the core is always included in the quasi-core, because the conditions defining the quasi-core are weaker.

A system $(\mathbf{s}, \pi)$, where $\mathbf{s}$ is an admissible state of $\mathfrak{E}$ and $\pi$ is a system of prices, is at equilibrium whenever agents of all types choose activities which result in the highest possible income and the allocation of commodities is consistent with the demand, i.e. for $i=1, \ldots, n$ we have

$$
s^{i}\left(\left\{(j, \mathbf{x}) \in W \mid r_{j}^{i} \pi_{j}=\max _{l \in V} r_{l}^{i} \pi_{l}, \mathbf{x}=d^{i}\left(r_{j}^{i} \pi_{j}, \pi\right)\right\}\right)=1 .
$$

If $(\mathbf{s}, \pi)$ is at equilibrium then the system $(\mathbf{p}, \pi)$ is at competitive equilibrium, where the distribution $\mathbf{p}=\left(p^{1}, \ldots, p^{n}\right)$ is given by $p_{j}^{i}=s^{i}\left(\{j\} \times \mathbb{R}_{+}^{k}\right)$ for $i=1, \ldots, n, j=1, \ldots, k$ (distributions of activities of agents of all types are induced by the state $\mathbf{s})$.

A state of the economy $\mathfrak{E}$ is an anonymous description of distribution of actions of all agents and of allocation of commodities. When dealing with the solutions like core and quasi-core we consider direct exchange within coalitions without taking into account any market mechanism resulting in a system of prevailing prices. On the other hand, we are interested in the actual consumption described by the allocation of commodities in a given state of $\mathfrak{E}$ and defined at competitive equilibrium by demand functions. Both descriptions of the economy - a system $(\mathbf{p} ; \pi) \in\left(\Delta_{k}\right)^{n+1}$ and a state $\mathbf{s}$ - involve the same information concerning the distribution of the agents' actions and thus the volume of aggregated supply. A state is admissible whenever the aggregated consumption is not greater than the aggregated production. The same condition is true at any competitive equilibrium. A coalition at a given state s may be informally understood as being included in the set of all agents of all types, choosing activities and consuming according to the description given by the state $\mathbf{s}$. An action available to a coalition is an anonymous description of changes in the decisions concerning the choice of activities made by agents in the coalition and of re-allocation of commodities within 
the coalition. An action is admissible to a coalition whenever the aggregated consumption within the coalition is provided by the aggregated production of the coalition's members. An action is profitable to a coalition if it results in improving the situation of the members of the coalition in the sense of increase of the value of their utility functions. The core is the set of all states of the economy $\mathfrak{E}$ such that there is no coalition for which there exists an action admissible and profitable as well. The quasi-core is the set of all states of the economy $\mathfrak{E}$ such that there is no coalition for which there exists an admissible and profitable action which involves only re-allocation of commodities within the coalition without any changes in agents' decisions concerning the production. Therefore, if any state belongs to the core, it is also in the quasi-core; the converse is usually not true.

3. Main results. We shall now prove two theorems determining relations of competitive equilibria, core and quasi-core.

TheOREM 1. If a state $\mathbf{s}$ belongs to the core of the economy $\mathfrak{E}$ and the utility functions $u^{i}$ are essentially increasing for $i=1, \ldots, n$, then there exists a system of prices $\pi \in \Delta_{k}$ such that the system $(\mathbf{p}, \pi)$ is at competitive equilibrium, where $p_{j}^{i}=s^{i}\left(\{j\} \times \mathbb{R}_{+}^{k}\right)$ for $i=1, \ldots, n$ and $j=1, \ldots, k$.

In order to prove Theorem 1 we use the results given in Roman (Ekes) and Wieczorek [6] and we prove a lemma concerning Pareto optimality of an aggregated supply in the core. First we quote a theorem proved in [6]:

Theorem 2. A distribution vector $\mathbf{p}=\left(p^{1}, \ldots, p^{n}\right)$ is weakly Pareto efficient if and only if there exists a system of prices $\pi=\left(\pi_{1}, \ldots, \pi_{k}\right) \in \Delta_{k}$ at which $p^{i}$ maximizes the total income of type $i$ equal to $q_{i} \sum_{j=1}^{k} r_{j}^{i} \pi_{j} p_{j}^{i}$, for each $i=1, \ldots, n$, and such that, for $j=1, \ldots, k, \pi_{j}>0$ if and only if there exists no distribution $\widetilde{\mathbf{p}}$ such that $S(\widetilde{\mathbf{p}})>S(\mathbf{p})$ and $S(\widetilde{\mathbf{p}})_{j}>S(\mathbf{p})_{j}$.

Now we shall prove the following lemma:

Lemma 3. If a state $\mathbf{s}$ belongs to the core of the economy $\mathfrak{E}$, then the aggregated supply in $\mathbf{s}$ is weakly Pareto optimal.

Proof of the lemma. Suppose that a state $\mathbf{s}$ is in the core. The aggregated supply in $\mathbf{s}$ is given by the formula

$$
\left(\sum_{i=1}^{n} q_{i} r_{1}^{i} s_{1}^{i}, \ldots, \sum_{i=1}^{n} q_{i} r_{k}^{i} s_{k}^{i}\right) .
$$

Set $p_{j}^{i}=s^{i}\left(\{j\} \times \mathbb{R}_{+}^{k}\right)$. Suppose that the vector of aggregated supply in $\mathbf{s}$ is not weakly Pareto optimal, i.e. there exists a distribution $\widetilde{\mathbf{p}}=\left(\widetilde{p}^{1}, \ldots, \widetilde{p}^{n}\right) \in$ 
$\left(\Delta_{k}\right)^{n}$ such that for all $j=1, \ldots, k$ we have

$$
\sum_{i=1}^{n} q_{i} r_{j}^{i} s_{j}^{i}<\sum_{i=1}^{n} q_{i} r_{j}^{i} \tilde{p}_{j}^{i}
$$

Consider the coalition $\boldsymbol{\sigma}=\mathbf{s}$ and the system of measures $m^{i}$ for $i=$ $1, \ldots, n$ defined by

$$
\begin{aligned}
m^{i}\left(\{j\} \times \mathbb{R}_{+}^{k} \times V \times \mathbb{R}_{+}^{k}\right) & =p_{j}^{i} \quad \text { for } j=1, \ldots, k, \\
m^{i}\left(V \times \mathbb{R}_{+}^{k} \times\{j\} \times \mathbb{R}_{+}^{k}\right) & =\widetilde{p}_{j}^{i} \quad \text { for } j=1, \ldots, k, \\
m^{i}\left(V \times X \times V \times \mathbb{R}_{+}^{k}\right) & =m^{i}\left(V \times \mathbb{R}_{+}^{k} \times V \times X\right) \\
& =s^{i}(V \times X) \quad \text { for all } X \in \mathfrak{B}\left(\mathbb{R}_{+}^{k}\right) .
\end{aligned}
$$

Then there exists an action $\mathbf{f}=\left(f^{1}, \ldots, f^{n}\right)$ such that $m^{i}=m_{\mathbf{f}}^{i}=\sigma^{i} \circ f^{i}$ for $i=1, \ldots, n$ (this follows from the Radon-Nikodym theorem).

We define

$$
x_{j}^{\prime}=x_{j}+\left(\sum_{i=1}^{n} q_{i} r_{j}^{i} \widetilde{p}_{j}^{i}-\sum_{i=1}^{n} q_{i} r_{j}^{i} p_{j}^{i}\right)\left(\sum_{i=1}^{n} q_{i}\right)^{-1} \quad \text { for } j=1, \ldots, k
$$

and $\mathbf{x}^{\prime}=\left(x_{1}^{\prime}, \ldots, x_{k}^{\prime}\right)$. Moreover, for $\mathbf{p} \in\left(\Delta_{k}\right)^{n}, j=1, \ldots, k$, we set

$$
\mathbf{X}_{j}(\mathbf{p})=\sum_{i=1}^{n} q_{i} r_{j}^{i} p_{j}^{i}, \quad \mathbf{X}(\mathbf{p})=\left(\mathbf{X}_{1}(\mathbf{p}), \ldots, \mathbf{X}_{k}(\mathbf{p})\right)
$$

We have

$$
\begin{aligned}
\sum_{i=1}^{n} q_{i} \int_{\mathbb{R}_{+}^{k}} \mathbf{x}^{\prime} m_{\mathbf{f}}^{i}\left(V \times \mathbb{R}_{+}^{k} \times\right. & \left.V \times d \mathbf{x}^{\prime}\right)=\sum_{i=1}^{n} q_{i} \int_{\mathbb{R}_{+}^{k}} \mathbf{x}^{\prime} s^{i}\left(V \times d \mathbf{x}^{\prime}\right) \\
& =\sum_{i=1}^{n} q_{i} \int_{\mathbb{R}_{+}^{k}}\left(\mathbf{x}+\frac{\mathbf{X}(\widetilde{\mathbf{p}})-\mathbf{X}(\mathbf{p})}{\sum_{i=1}^{n} q_{i}}\right) s^{i}(V \times d \mathbf{x}) \\
& \leq \mathbf{X}(\mathbf{p})+\mathbf{X}(\widetilde{\mathbf{p}})-\mathbf{X}(\mathbf{p})=\mathbf{X}(\widetilde{\mathbf{p}})
\end{aligned}
$$

We also have

$$
m_{\mathbf{f}}^{i}\left(\left\{\left((j, \mathbf{x}),\left(j^{\prime}, \mathbf{x}^{\prime}\right)\right) \in W \times W \mid u^{i}\left(\mathbf{x}^{\prime}\right)>u^{i}(\mathbf{x})\right\}\right)=1 \quad \text { for } i=1, \ldots, n,
$$

since $\mathbf{x}^{\prime} \gg \mathbf{x}$ and all utility functions $u^{i}$ are essentially increasing.

Therefore, given a state $\mathbf{s}$, we have found a coalition which has an admissible and profitable action. It follows that $\mathbf{s}$ is not in the core, which is a contradiction.

Proof of Theorem 1. If a state $\mathbf{s}$ belongs to the core of $\mathfrak{E}$ then by Lemma 3 the aggregated supply $S(\mathbf{p})$ is weakly Pareto optimal for $\mathbf{p}=\left(p^{1}, \ldots, p^{n}\right)$ and $p_{j}^{i}=s^{i}\left(\{j\} \times \mathbb{R}_{+}^{k}\right)$ for all $i=1, \ldots, n$ and $j=1, \ldots, k$. Theorem 2 implies that the distributions $p^{i}$ maximize the aggregated income of agents 
of type $i$, for $i=1, \ldots, n$, for some system of prices $\pi$. If the distribution $p^{i}$ maximizes the aggregated income of agents of type $i$ then the individual income $r_{j}^{i} \pi_{j}$ of agents of this type is the same for all agents of type $i$ and equal to $\max _{l=1, \ldots, k} r_{l}^{i} \pi_{l}$ unless $p_{j}^{i}=0$. If not, that is, if for some $j$ we had $p_{j}^{i} \neq 0$ and $r_{j}^{i} \pi_{j}<\max _{l=1, \ldots, k} r_{l}^{i} \pi_{l}$, then we could take a distribution $\widetilde{p}^{i}$ such that $\widetilde{p}_{j}^{i}=1$ for $j \in \operatorname{Argmax}_{l=1, \ldots, k} r_{l}^{i} \pi_{l}$, which would yield a contradiction, since $q_{i} \sum_{j=1}^{k} r_{j}^{i} \pi_{j} p_{j}^{i}<q_{i} \sum_{j=1}^{k} r_{j}^{i} \pi_{j} \widetilde{p}_{j}^{i}$. The conclusion is that at prices $\pi$ and given distribution $\mathbf{p}$ of decisions of agents, agents of all types maximize their individual income and the aggregated supply is not less than the aggregated demand (which follows from the equality $\left\langle d^{i}(I, \pi) ; \pi\right\rangle=I$ ). Therefore the system $(\mathbf{p}, \pi)$ is at competitive equilibrium.

THEOREM 4. If a system $(\mathbf{s}, \pi)$ is at equilibrium then the state $\mathbf{s}$ belongs to the quasi-core of the economy $\mathbb{E}$.

Proof. Suppose that $\mathbf{s}$ is at equilibrium with $\pi$ but not in the quasi-core of $\mathfrak{E}$. Then, for $i=1, \ldots, n$, there exist measures $m^{i}$ on $W \times W\left(m^{i}=\sigma^{i} \circ f^{i}\right)$ satisfying the following conditions (for the notation $V_{j}$, see Section 2):

$$
\begin{aligned}
& \sum_{i=1}^{n} q_{i} \int_{\mathbb{R}_{+}^{k}} \mathbf{x}^{\prime} m^{i}\left(V \times \mathbb{R}_{+}^{k} \times V \times d \mathbf{x}^{\prime}\right) \\
& \quad \leq\left(\sum_{i=1}^{n} q_{i} r_{1}^{i} m^{i}\left(V_{1}\right), \ldots, \sum_{i=1}^{n} q_{i} r_{k}^{i} m^{i}\left(V_{k}\right)\right) \\
& m^{i}\left(\left\{\left((j, \mathbf{x}),\left(j^{\prime}, \mathbf{x}^{\prime}\right)\right) \in W \times W \mid u^{i}\left(\mathbf{x}^{\prime}\right)>u^{i}(\mathbf{x})\right\}\right)=m^{i}(W \times W) ; \\
& \sum_{j=1}^{k} m^{i}\left(\{j\} \times \mathbb{R}_{+}^{k} \times\{j\} \times \mathbb{R}_{+}^{k}\right)=m^{i}(W \times W) .
\end{aligned}
$$

Since the pair $(\mathbf{s}, \pi)$ is at equilibrium, all agents of the same type have the same income. We denote the income of agents of type $i$ by $I^{i}$.

We may write condition (2) in the following way:

$$
\begin{aligned}
& m^{i}\left(\left\{\left(\left(j, d^{i}\left(I^{i}, \pi\right)\right),\left(j, \mathbf{x}^{\prime}\right)\right) \in W \times W \mid\right.\right. \\
& \left.\left.u^{i}\left(\mathbf{x}^{\prime}\right)>\max \left\{u^{i}(\mathbf{x}) \mid \mathbf{x} \in \mathbb{R}_{+}^{k},\langle\pi ; \mathbf{x}\rangle \leq I^{i}\right\}\right\}\right)=m^{i}(W \times W)
\end{aligned}
$$

(by the definition of equilibrium and (2), (3)). Therefore we must have $\left\langle\pi ; \mathbf{x}^{\prime}\right\rangle>I^{i}$ for $i=1, \ldots, n$, and then

$$
\begin{aligned}
\sum_{i=1}^{n} q_{i} \int_{\mathbb{R}_{+}^{k}}\left\langle\pi ; \mathbf{x}^{\prime}\right\rangle m^{i}\left(V \times \mathbb{R}_{+}^{k} \times V\right. & \left.\times d \mathbf{x}^{\prime}\right) \\
& >\sum_{i=1}^{n} q_{i} \int_{\mathbb{R}_{+}^{k}} I^{i} m^{i}\left(V \times \mathbb{R}_{+}^{k} \times V \times d \mathbf{x}^{\prime}\right) .
\end{aligned}
$$

Therefore 


$$
\left\langle\pi ; \sum_{i=1}^{n} q_{i} \int_{\mathbb{R}_{+}^{k}} \mathbf{x}^{\prime} m^{i}\left(V \times \mathbb{R}_{+}^{k} \times V \times d \mathbf{x}^{\prime}\right)\right\rangle>\sum_{i=1}^{n} q_{i} I^{i} m^{i}\left(V \times \mathbb{R}_{+}^{k} \times V \times \mathbb{R}_{+}^{k}\right) .
$$

Using (1) we may write

$$
\begin{aligned}
\left\langle\pi ; \sum_{i=1}^{n} q_{i} \int_{\mathbb{R}_{+}^{k}} \mathbf{x}^{\prime} m^{i}\left(V \times \mathbb{R}_{+}^{k}\right.\right. & \left.\left.\times V \times d \mathbf{x}^{\prime}\right)\right\rangle \\
& \leq\left\langle\pi ;\left(\sum_{i=1}^{n} q_{i} r_{1}^{i} m^{i}\left(V_{1}\right), \ldots, \sum_{i=1}^{n} q_{i} r_{k}^{i} m^{i}\left(V_{k}\right)\right)\right\rangle \\
& =\sum_{i=1}^{n} q_{i} \pi_{1} r_{1}^{i} m^{i}\left(V_{1}\right)+\ldots+\sum_{i=1}^{n} q_{i} \pi_{k} r_{k}^{i} m^{i}\left(V_{k}\right) \\
& =\sum_{i=1}^{n} q_{i} I^{i} m^{i}\left(V \times \mathbb{R}_{+}^{k} \times V \times \mathbb{R}_{+}^{k}\right),
\end{aligned}
$$

which yields a contradiction.

4. Final remarks. It is natural to make a difference between the two solutions: core and quasi-core in the application considered. The core is a set of states at which nobody can improve the situation by changing the decision concerning the produced commodity and/or by re-allocating the commodities. The quasi-core includes those states at which there is no possibility for any set of agents to improve their situation only by reallocating the goods, the distribution of decisions being settled. There is a kind of asymmetry in defining states of economy by means of distribution of agents' decisions and demand functions and by means of distributions on the product space of decisions and commodities. In the first approach agents do not decide on the allocation of commodities; they get the bundles of commodities assigned by their demand function (unless the total demand exceeds the total supply). This is the reason why in Theorem 1 we use only the distribution of commodities generated by the state $\mathbf{s}$. If we have the distribution of decisions of all agents and a system of prices, we know the whole state of the economy in the first approach. Agents are maximizing their income by deciding on which commodity to produce and this results in the maximization of their consumption (the value of the demand function). On the other hand, in Theorem 4 we show that if a system $(\mathbf{s}, \pi)$ is at equilibrium then no coalition can improve the situation of its members by changing the prevailing allocation, which assigns to each agent the value of the demand function, i.e. the state is in the quasi-core.

The model of household economy may be reduced to a noncooperative game with a continuum of players classified into a finite number of types, called a large game of finite type (see Wieczorek [8]). Players of all types 
have finite sets of available strategies and they are characterized by their payoff functions on the product of the set of individual strategies and the set of all sequences of distributions of individual decisions of all types of players. Players of the same type are characterized by the same payoff function. A sequence of distributions of players' decisions is at equilibrium whenever no player wants to change the chosen strategy, provided that all remaining players stick to their choices. If we reduce the model of household economy to a large game of finite type, we assume that all players, representing agents of various types, have the same set of strategies containing the given activities and the payoff is equal to the individual income. We also introduce one more type of players, representing the market mechanism clearing the market by setting the prices.

Acknowledgements. I am grateful to the referee whose remarks helped to improve the paper. I also wish to thank Andrzej Wieczorek for many useful comments.

\section{References}

[1] R. Aumann, Markets with a continuum of traders, Econometrica 32 (1964), 39-50.

[2] - Existence of competitive equilibria in markets with a continuum of traders, ibid. 34 (1966), 1-17.

[3] M. Ekes, Models of economy with infinitely many consumers and producers classified into a finite number of types, PhD thesis, Warszawa, 2000 (in Polish).

[4] W. Hildenbrand, Core and Equilibria of a Large Economy, Princeton Univ. Press, 1974.

[5] A. Mas-Colell, On a theorem of Schmeidler, J. Math. Econom. 13 (1984), 201-206.

[6] M. Roman (Ekes) and A. Wieczorek, On a multi-objective optimization problem arising from production theory, Appl. Math. (Warsaw) 25 (1999), 411-415.

[7] K. Vind, Edgeworth-allocations in an exchange economy with many traders, Internat. Econom. Rev. 5 (1964), 165-177.

[8] A. Wieczorek, Elementary large games and an application to economies with many agents, Inst. Computer Sci., Polish Acad. Sci. Report 805, Warszawa, 1996.

[9] - Household economies with infinitely many agents: a game-theoretic analysis, Internat. Game Theory Rev. 4 (2002), 319-329.

Institute of Econometrics

Warsaw School of Economics

Al. Niepodległości 164

02-554 Warszawa, Poland

E-mail: maria.ekes@sgh.waw.pl
Institute of Computer Science

Polish Academy of Sciences

Ordona 21

01-237 Warszawa, Poland

Received on 10.10.2002;

revised version on 14.5.2003 